\title{
The Phase Detection Algorithm of Weak Signals Based on Coupled Chaotic-oscillators
}

\author{
Sun Wen Jun, Rui Guo Sheng, Zhang Yang \\ School of Naval Aeronautical and Astronautical University, Yantai 264001, China
}

Keywords: phase detection; coupled oscillators; state stability; noise immunity

\begin{abstract}
Traditional phase detection methods of weak signals have some problems such as high complexity and low accuracy. To cope with these problems,, this paper proposes one novel parallel detection algorithm based on multi-coupled oscillators. Firstly, the chaotic detection model and double coupled oscillator model are studied through EM algorithm. And then, the nonlinear dynamics are studied in-depth, and the noise immunity and stability analyzes of the algorithm are proved in paper. Finally, the weak harmonic power carrier signals simulation results are demonstrated for the effectiveness of the algorithm.
\end{abstract}

\section{Introduction}

Known as one of the most important theories in the 20th century, Chaotic algorithm has been widely used in many fields such as signal detection and estimation, neural network control and so on [1-4]. It is a complex mathematical process for using nonlinear chaotic oscillator to the process of weak signal detection. Especially, the key technology of the algorithm is detection of weak signal's initial phase [5].

At present, domestic and foreign scholars have made a lot of research work on the method of coupled oscillators. But, there are still some problems. The double-coupled oscillator is introduced as detection model in literature [6] and [7], but the studies lack the in-depth analysis of system dynamics and robustness.

\section{System Model}

\subsection{Principle of chaotic system model}

The common model of chaotic dynamical systems is composed of Duffing oscillator, Lorenz oscillator, Hemon oscillator and so on $[9,10]$. Respectively, there is most fully research and application about classical Duffing oscillator. And hence, the classical Duffing system is introduced as detection model:

$$
\ddot{x}(t)+k \dot{x}(t)-x(t)+x^{3}(t)=A(t)+n(t),
$$

where $A(t)=\cos (\omega t)$ is the cycle driving force, $k$ is the damping ratio, $u=-x+x^{3}$ is the non-linear restoring force, and $n(t)$ is the white noise. The combined driving force could be rewritten as follows:

$$
\begin{aligned}
A(t) & =\lambda_{d} \cos (\omega t)+a \cos (\omega t+\phi) \\
& =\lambda(t) \cos (\omega t+\theta(t))
\end{aligned},
$$

where $\lambda_{d}$ is the critical threshold of system, $a$ is the amplitude of to-be-detected signals. At this time, the overall amplitude of excitation signal can be described as:

$$
\lambda=\sqrt{\lambda_{d}^{2}+2 \lambda_{d} \cos (\phi)+a^{2}} .
$$

The overall phase of excitation signal can be described as:

$$
\theta=\operatorname{arctg} \frac{a \sin (\phi)}{\lambda_{a}+a \cos (\phi)}
$$

The specific relationship between system states and the phase of to-be-detected signals can be written as follows: 


$$
\pi-\arccos \left(a / 2 f_{d}\right) \leq \phi \leq \pi+\arccos \left(a / 2 f_{d}\right) .
$$

When the initial phase of to-be-detected signals could satisfy the mapping function above, the system continues to be in chaotic state. It does not occur an transition change from chaotic state to large-scale periodic state. On the contrary, system state changes into large-scale periodic state, encouraged by the excitation signal.

\subsection{Analysis of coupled oscillators model}

The double coupled oscillator system model is written as follows:

$$
\left\{\begin{array}{l}
\ddot{x}(t)+k \dot{x}(t)+\varepsilon-u=\lambda \cos (\omega t) \\
\ddot{u}(t)+k \dot{u}(t)+\varepsilon-u=\lambda \cos [(\omega+\Delta \omega) t+\varphi]
\end{array},\right.
$$

where the coupling term is $\varepsilon=c k(x(t)-u(t))$.

The system model is one second-order stochastic differential equation and the exact analytical solution is not available by traditional methods. In addition, the double coupled system is rather sensitive to the initial value of to-be-detected signals. If the numerical solution accuracy of differential equations is not suitable, the characteristics of chaotic system could not be described accurately. Therefore the EM algorithm (Euler-Maruyama) is introduced, and the system model could be rewritten as follows:

$$
\left\{\begin{array}{l}
x_{\mathrm{k}+1}=h y_{k} \\
y_{k+1}=\left(-k y_{k}+x_{k}-x_{k}^{3}+\lambda \cos \left(\omega t_{k}\right)\right) h+\sqrt{2 D} \cdot \sqrt{h} \sigma_{k}
\end{array}\right.
$$

where $h$ is the integration step, $\sigma_{k}$ is the Gaussian random sequence with zero-mean value and unit variance.

\section{Characteristic analysis of Coupled System Model}

\subsection{Analysis of system dynamics}

When the initial phase of cycle driving force built in system changes, the bifurcation value of double coupled oscillator varies from the pristine one.

To study the system fractal characteristics, simulation experiments are designed. The main parameters are set as follows: the frequency of cycle driving force built in system is $\omega=1 \mathrm{rad} / \mathrm{s}$, and the integration step is $h=1 / 100$, the range of initial phase is from $-\pi / 4$ to $\pi / 6$. The results compared with two different models between initial phase and bifurcation values are shown in Tab. 1.

Table 1 bifurcation values of single and coupled model with different initial phase

\begin{tabular}{cccc}
\hline Initial phase & $-3 \pi / 12$ & $-2 \pi / 12$ & $-1 \pi / 12$ \\
\hline Single oscillator & 0.82489 & 0.82514 & 0.82421 \\
Coupled oscillator & 0.82561 & 0.82534 & 0.82476 \\
\hline Initial phase & 0 & $1 \pi / 12$ & $2 \pi / 12$ \\
\hline Single oscillator & 0.82560 & 0.82614 & 0.82456 \\
Coupled oscillator & 0.82550 & 0.82632 & 0.82514 \\
\hline
\end{tabular}

From Tab. 1, we can get a conclusion that the initial phase of cycle driving force built in system is influential to the system fractal characteristics. Therefore, in the actual signal detection experiments, the phase of excitation signal should be determined according to the initial phase of to-be-detected signals. However, compared with classic single oscillator, the bifurcation value of coupled oscillator system represents more stable, the affection generated by the preset phase is much smaller. These reasons are conducive for the actual signals detection.

\subsection{Analysis of system state stability}

To study the system state stability, simulation experiments are designed. The main parameters are set similar to the former chapter. Afterwards, with the false alarm and missed alarm probability as detection index, the results under chaotic state and large-scale periodic state are studied by simulation experiments.

Both single and double coupled oscillator systems are adjusted to achieve the critical chaotic 
state, and the false alarm probability under the background noise in different intensity. The strength range of to-be-detected signals is from 0.001 to 0.01 , the background noise generated by the noise generator with different intensities is injected into the test system. There are 100 times of Monte Carlo simulation experiments, each time 10000 valid data is selected for the system state stability judging. The false alarm and missed alarm probabilities obtained through experiments are shown in Tab. 2.

Table. 2 Detection performance under noise of different strength

\begin{tabular}{cccc}
\hline & $\begin{array}{c}\text { Noise } \\
(\mathrm{W})\end{array}$ & $\begin{array}{c}\text { Single } \\
\text { oscillator }\end{array}$ & $\begin{array}{c}\text { Coupled } \\
\text { oscillator }\end{array}$ \\
\hline & 0.001 & 0.002 & 0 \\
false alarm & 0.002 & 0.004 & 0.001 \\
(critical state) & 0.005 & 0.022 & 0.012 \\
& 0.01 & 0.051 & 0.032 \\
\hline missed alarm & 0.001 & 0.001 & 0 \\
(periodic & 0.002 & 0.003 & 0 \\
state) & 0.005 & 0.017 & 0.007 \\
& 0.01 & 0.043 & 0.026 \\
\hline
\end{tabular}

From Tab. 1, we can get a conclusion that with the strength of background noise increasing, the false alarm and missed alarm probabilities of single and coupled oscillator systems gradually become larger. However, the false alarm and missed alarm probabilities of two coupled oscillators are always lower than single oscillator. The results indicate that the proposed model is more stable, more conducive to the accurate detection and synchronize of weak signals' initial phase under strong noise background.

\subsection{Analysis of noise immunity}

The noise immunity of system is most important to the phase states discrimination. Therefore simulation experiments are designed to study the capability of both single and double coupled oscillator systems.

Both single and double coupled oscillator systems are adjusted to achieve the critical chaotic state, compare with the track circumstances at the same noise strength. Te results obtained by simulation are shown in Fig. 2. The main parameters are set similar to the first chapter, and the noise power is $\sigma=0.001 \mathrm{~W}$.

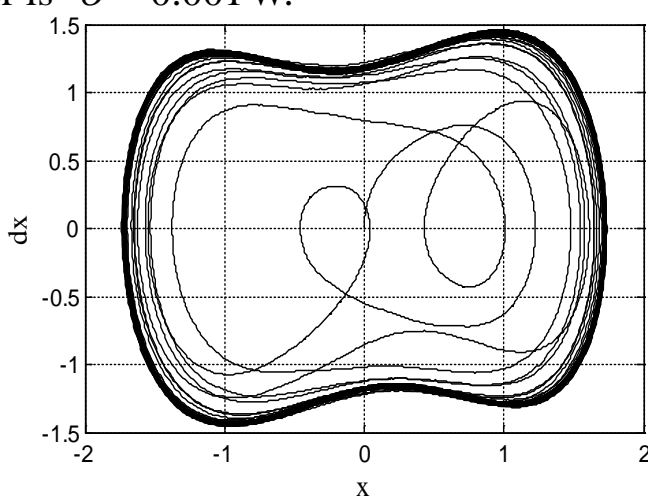

(a) Single Duffing oscillator

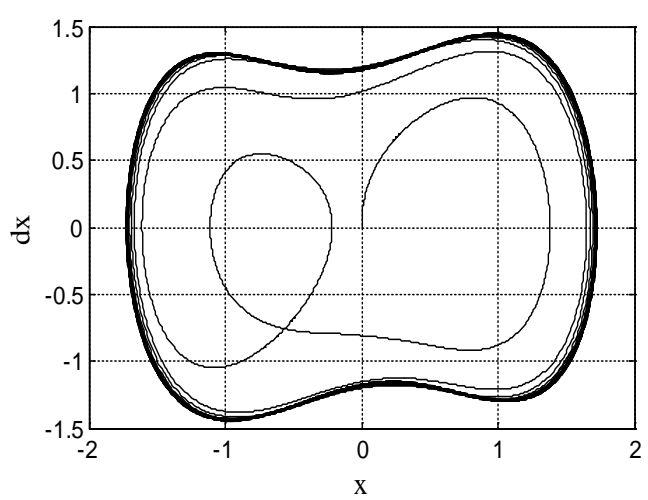

(b) Double coupled oscillato

Fig. 2 Track circumstances of single and double coupled oscillator systems

From Fig. 2, we can see that at the same noise strength, compared with classic Duffing oscillator, double coupled oscillator has always maintained one better working condition, and the speed transforming from chaotic state into large-scale periodic state is much faster. The results indicate that the proposed system has much more excellent noise immunity.

\section{Simulation Experiments}

To study the effectiveness of coupled oscillator model application in phase detection of weak signals. This section uses double coupled oscillators array to study the initial phase detection of weak harmonic power carrier signals. 
However, according to the state determination algorithm and the restriction of chaotic oscillators, the algorithm accuracy and phase detection range are determined by the strength of to-be-detected signals. the sensitivity of initial phase could not achieve the accuracy and range of theoretical detection. Therefore multi-oscillator array is designed to make up this deficiency. The phase range of $[-\pi, \pi]$ is divided in different parts such as $\{-k \pi / n, \cdots, 0, \cdots, k \pi / n\}$, in which $k=0,1, \cdots n$.

The multi-oscillator array composed of coupled oscillatot is introduced in experiments. The main parameters are set as follows: the damping ratio of system $k=0.5$, the integration step is $h=0.01 \mathrm{~s}$, the frequency of cycle driving force built in system is $\omega=1 \mathrm{rad} / \mathrm{s}$, the simulation time is $1000 \mathrm{~s}$. The detection object is 1000 troops of sinusoidal signals with random initial phase. The scope range of initial phase is $[-\pi, \pi]$, and the detection error results are shown in Fig. 3.

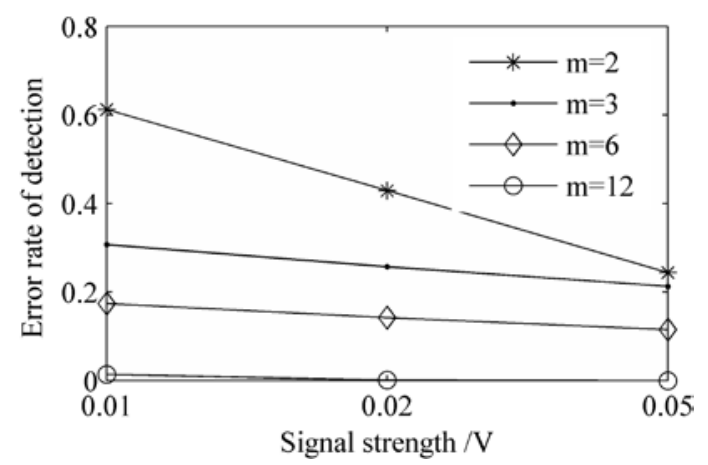

Fig. 3 Detection error results under noise of different strength

From Fig.3, we can get a conclusion that with the enhance of the excitation signal strength, the number of multi-oscillator array required is also reduced. In addition, with the increment of array number, the detection error is also reduced quickly. The experiment results indicate that the proposed parallel detection array based on multi-coupled oscillators is available for the phase detection of weak signals.

\section{Summary}

Traditional phase detection methods of weak signals have some problems such as high complexity and low accuracy. To cope with the problems, one novel parallel detection algorithm based on multi-coupled oscillators is introduced in paper. Firstly, the chaotic detection model and double coupled oscillator model are studied through EM algorithm. On this basis, the system dynamics, system state stability and noise immunity are analyzed in-depth. Finally, the weak harmonic power carrier signals are employed to verify the effectiveness of proposed algorithm. Experiment results indicate that the robustness of proposed algorithm is better than traditional single oscillator, the anti-noise performance is strong enough for the phase detection of weak signals. The proposed algorithm provides a new way for the phase detection of weak signals, and has one certain theoretical meaning and practicable value.

\section{References}

[1] H Chen, P K Varshney, S M Kay, and J H Michels. Theory of stochastic rensonance effect in signal detection part1: dixed detectors[J]. IEEE transactions on Signal Processing, 2005, 55(7):3172-3185.

[2] Li Yue, Shi Yao-wu, Ma Hai-tao, et al. Chaotic detection method for weak square wave signal Submerged in colored noise[J]. Acta Electronica Sinica, 2004,32(1): 87-90.

[3] Fan Jian, ZhaoWen-li, and Wang Wan-qiang. Study on the weak sinusoidal signal detection property using Duffing chaos system[J]. Acta Physis Sinica, 2013:45(1):1-7.

[4] Mloon F, Tayle G, Diler B, et al.. Pseudorandom number generator based on mixing of three chaotic maps[J]. Communications in Nonlinear Science and Numerical Simulation, 2014,19(4):1-8. 
[5] Zhang Shu-qing, Zhai Xin-pei, Dong Xuan, et al.. Application of EMD and Duffing oscillator to fault line detection in un-effectively grounded system[J]. Proceedings of the CSEE, 2013,33(10):161-167.

[6] Yang J, Xiong Y P, Xing J T. Nonlinear power flow analysis of the Duffing oscillator[J]. Mechanical Systems and Signal Processing, 2014,45(2):563-578.

[7] Nie Chun-yan, and Shi Yao-wu. The research of weak signal detection based on cross-orrelation and chaos theory[J]. Chinese Journal of Scientific Instrument, 2001,22(1):32-35.

[8] Sun Zi-qiang, Chen Chang-zheng, Gu Yan-ling, et al. Incipient fault diagnosis of large scale wind turbine gearbox based on chaos theory and sampling integral technology[J]. Journal of Vibration and Shock, 2013, 32(9): 113-117.

[9] Dimitrios E P, Efstathios E T and Michalis P M. Exact analytic solutions for the damped Duffing nonlinear oscillator[J]. Chaos, Solitons and Fractals, 2006(334): 311-316.

[10]Zhu H T. Probabilistic solution of vibro-impact stochastic Duffing systems with a unilateral non-zero offset barrier[J]. Physica A: Statistical Mechanics and its Applications, 2014,410:335-344.

[11]Boudra A O, Cexus J C. IF estimation using empirical mode decomposition and nonlinear Teager energy operator[C]. IEEE First International Symposium on Control Communications and Signal Processing. 2004, 45-48.

[12]Liu Hai-bo, Wu De-wei, Jin Wei, et al.. Syudy on weak signal detection method with Duffing oscillators[J]. Acta Physis Sinica, 2013, 62(05):42-47. 